\title{
Role of Autologous Adipose derived Mesenchymal Stem Cells and Platelet Rich Plasma in Knee Osteoarthritis
}

\author{
Anant Marathe, Jaymesh Thadani ${ }^{1}$, Prashant Kshatriya, Shivani Vakodikar, Rajni Vyas \\ Total Potential Cells (P) Ltd; 'Department of Zoology, Faculty of Science, The M.S.University of Baroda, Vadodara, Gujarat; India.
}

\section{Corresponding Author:}

Dr. Anant Marathe

Email: dranantmarathe@hotmail.com

This is an Open Access article distributed under the terms of the Creative Commons Attribution License (creativecommons.org/ licenses/by/3.0).

Received Accepted Published

August 15, 2017

October 13, 2017

November 25, 2017

\begin{abstract}
Background: Conventional treatments of osteoarthritis demonstrate only modest clinical benefits without lesion reversal. Autologous mesenchymal stem cells (MSCs) are known to have potential for articular cartilage regeneration. We assess the feasibility and safety of treating osteoarthritis autologous MSCs in combination with platelet rich plasma (PRP). Material \& Methods: Patients with chronic knee pain unresponsive to conservative treatments and showing radiological evidence of osteoarthritis were enrolled and injected adipose derived mesenchymal stem cells intra-articularly. The MSC-treated patients displayed significant improvement with Western Ontario and McMaster Universities Osteoarthritis index (WOMAC) score. No adverse events related local or systemic were found. Conclusion: Autologous MSC therapy may be a valid alternative for the treatment of chronic knee osteoarthritis.
\end{abstract}

Keywords: Intra-articular Injections, Regeneration, Knee Osteoarthritis, Mesenchymal Stromal cells, Pain.

\section{Introduction}

Osteoarthritis is the most prevalent chronic joint disease and a frequent cause of joint pain and disability [1]. Unfortunately, conventional treatments demonstrate only modest clinical benefits [2] and articular replacement by prosthesis is recommended only as a last treatment option. Cell-based therapies are promising for the treatment of osteoarthritis and have shown encouraging results in animal studies and in human case reports.

Mesenchymal stem cells (MSCs) have currently gained wide attention in therapeutics as they have properties to regenerate articular cartilage defects [3]. Stem cells are immature precursor cells possessing the capacity for self-renewal. They are immunonaive and multi-lineage [4], with proven chondrogenic translation, in vivo [5]. Many animal studies [6] and, recent human studies have documented that MSCs initiated regeneration of cartilage defects. It is well documented that platelet rich plasma (PRP) has anti-inflammatory properties $[7,8]$.

We present evidence to suggest that intra-articular injection of autologous MSCs has therapeutic value for treating knee osteoarthritis. ADMSCs are having high yield of MSCs and BMSCs from aged patient produced low amount of mineral deposits and had a lower expression level of osteogenic genes [9].

\section{Materials and Methods}

The present study was carried out after approvals from Institutional Ethics Committee and Institutional Committee for Stem Cell Research, Total Potential Cells (P) Ltd, Vadodara. The study was enrolled as a clinical trial CTRI No 2013/02/004619. A written consent from patients was obtained after discussion. 
Patients with either sex with age between 55 to 70 years; near normal body mass index and osteoarthritis (OA) diagnosis based on radiological evidence were included. Patients with metabolic disorder such as hypothyroid, diabetes or abnormal blood pressure levels were controlled before the initialization of treatment. Assessment of WOMAC score of each enrolled subject was done. Patients with history of taking corticosteroids or NSAIDs, glycosaminoglycans, or suffering from active cardiac or respiratory disease; Hepatitis B, C, or HIV positive; and patients with history of allergic reactions were excluded from the study. Patients with loose bodies in the joint were excluded.

A total of six patients, four female and two male with radiological evidence of knee OA that would have necessitated joint replacement surgery and satisfying the above mentioned criteria were selected for the study. The patients underwent blood investigation before commencement of therapy. This included complete blood count, blood sugar, blood urea, serum creatinine, SGPT, HIV (I and II) antibody test, hepatitis B, surface antigen \& HCV antibody test, prothrombin time, thyroid stimulating hormone estimation were done to ascertain fitness of treatment.

Adipose tissue was aspirated by a trained plastic surgeon with specially designed aspiration cannulae under local anaesthesia and sedation. Lipoaspirate was collected from lower abdominal subcutaneous fat in the operating room in a sterile container. The samples were transferred to a clean room for cell isolation under strict aseptic conditions. The isolation of mesenchymal stem cells was performed with density gradient centrifugation technique. Isolation was performed according to institutional protocol (patent pending). ADMSCs from lipoaspirate were isolated after digestion with $0.2 \%$ collagenase (Gibco Life Technologies, U.S.A, catalog no: 17100-17) in $\mathrm{CO}_{2}$ Incubator (Thermo Scientific, Forma Series II, Germany) at $37^{\circ} \mathrm{C}$ with gentle shaking. Cells obtained were made into a pellet at $1500 \mathrm{~g}$ in centrifuge (Remi Biotech,
Mumbai, India). Cells were further washed twice with D PBS and finally with Isolyte M (Clarias Ostuka, Ahmedabad, India). The cells were then seeded in the T-25 flask with high glucose media (HiMedia, catalog no: AL007S). The media change was done on every third day. The trypsinization (TrypLE - Gibco, catalog no: 12605 -028) was done once the confluency is attained. The cell counting is done with trypan blue dye.

The isolated ADMSCs were subjected to characterization for CD 29, CD 44, CD 90, CD 105 (positive markers) and CD 34, CD 45, HLA-DR (negative markers). Briefly, cells were fixed with freshly prepared $4 \%$ paraformaldehyde (PFA) for 10 minutes at room temperature and permeabilized with $0.1 \%$ Triton X-100 for 3-5 minutes on ice. Primary antibodies were incubated overnight at $4^{\circ} \mathrm{C}$, washed with PBS and then incubated with the secondary antibodies (Donkey anti-rabbit Alexa Flour 488; Jacksons Immunoresearch, United States) at room temperature for $1 \mathrm{~h}$. Slides were washed in PBS and mounted with Vectashield (Vector-Laboratories, CA, United States). DAPI (4, 6-diamidoino-2-phenylindole) (Invitrogen, United States) was used to visualize nuclei. Finally, cells were examined and Images were captured using fluorescent microscope.

Isolated cells were then suspended in $2 \mathrm{ml}$ of Isolyte M (Clarias Ostuka, Ahmedabad, India). PRP was obtained by the standard protocol by Graham, 2002. [8,10]. About $8 \mathrm{cc}$ of autologous PRP was obtained after centrifugation of $40 \mathrm{cc}$ heparinized blood. $4 \mathrm{ml}$ PRP (approximately $2,00,000$ platelet count/ $\mu \mathrm{l}$ ) was added to the isolated cell suspension. This was freshly injected in each knee joint intra articularly at 2 million cells/ $\mathrm{kg}$ body weight for one knee joint.

A radiological assessment after treatment was avoided because irradiation initiates apoptosis of human ADMSCs [11]. Arthroscopic evaluation of the regeneration of cartilage was not approved by the Ethics Committee. It was considered to be 
unnecessary in highly satisfied patients. Necessary follow up of patient were done and actual status of the knee was assessed by WOMAC [11] Index. This index evaluates pain with 5 criteria, stiffness with 2 criteria and physical function with 17 criteria. They are scored from 0-4 range. The scores are summed for items in each subscale, with possible ranges as follows: pain $=0-20$, stiffness $=0-8$, physical function $=0-68$, and a score is arrived.

\section{Results}

After liposuction of adipose tissue from lower abdomen, the thick viscous honey like lipoaspirate is obtained. The ADMSCs adhered on T- 25 flask looks like spindle shaped. They form compact structure which helps to reduce the friction between tibia and femur. Morphologically, the isolated cells were phenotype of a typical MSCs. Characterization with positive markers CD 29, CD 44, CD 90, CD 105 [Fig.1] and negative markers CD 34, CD 45, HLA-DR [Fig.2] was successfully done and verified under fluorescent microscope.

The most remarkable change following the treatment was immediate relief to total disappearance of pain within 24-72 hours. Similarly other parameters in WOMAC score also showed immediate improvement. No treatment related specific adverse events were observed in the patients after the induction in the trial. After follow up of one year, results demonstrated that dosage of 1-2 million cells/ $\mathrm{kg}$ body weight per knee is safe as well as effective in treating osteoarthritis. Table 1 represents decrease in WOMAC score which signifies improvement in joint functioning and patients were completely satisfied at the end of the study. Data are presented as mean $\pm \mathrm{SD}$ in Table 2. The $P$ value of less than 0.05 was considered statistically significant. Non-parametric using Welch T Test was used to analyze statistical differences between preoperative and followup values of WOMAC scores using Graph Pad Prism software, version 3.0 (GraphPad Software,

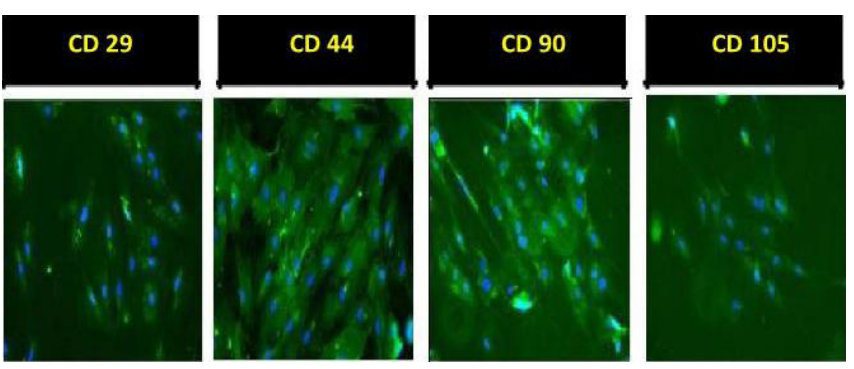

Fig.1: ADMSCs immunostained positively for CD29, CD44, CD90 and CD105.

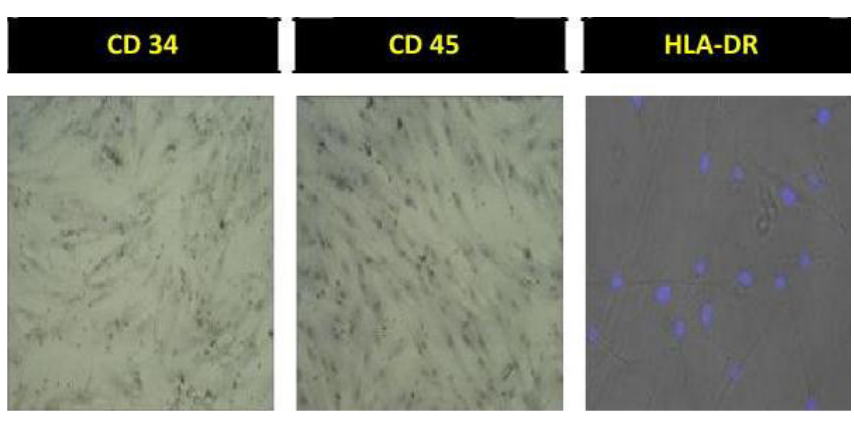

Fig.2: ADMSCs immunostained negative for CD34, CD45 and $H L A-D R$

Table 1: WOMAC Index pre and post treatment.

\begin{tabular}{|c|c|c|c|c|c|c|c|c|c|c|c|c|}
\hline WOMAC & \multicolumn{2}{|c|}{ Patient1 } & \multicolumn{2}{|c|}{ Patient2 } & \multicolumn{2}{|c|}{ Patient3 } & \multicolumn{2}{|c|}{ Patient4 } & \multicolumn{2}{|c|}{ Patient 5 } & \multicolumn{2}{|c|}{ Patient6 } \\
\hline & Pre & Post & Pre & Post & Pre & Post & Pre & Post & Pre & Post & Pre & Post \\
\hline Pain & 18 & 1 & 16 & 0 & 20 & 0 & 20 & 1 & 17 & 0 & 18 & 1 \\
\hline Stiffness & 7 & 1 & 6 & 1 & 6 & 0 & 8 & 1 & 7 & 0 & 6 & 1 \\
\hline $\begin{array}{c}\text { Difficulty in } \\
\text { Physical } \\
\text { functioning }\end{array}$ & 61 & 7 & 58 & 6 & 60 & 3 & 63 & 8 & 59 & 5 & 62 & 9 \\
\hline Total & 86 & 9 & 80 & 7 & 86 & 3 & 91 & 10 & 83 & 5 & 86 & 11 \\
\hline
\end{tabular}

Table 2: WOMAC Index pre and post treatment.

\begin{tabular}{|c|c|c|}
\hline $\begin{array}{c}\text { Statistical } \\
\text { Analysis }\end{array}$ & $\begin{array}{c}\text { Pre therapy Mean } \pm \\
\text { SD }\end{array}$ & $\begin{array}{c}\text { Post therapy Mean } \pm \\
\text { SD }\end{array}$ \\
\hline Pain & $18.16 \pm 1.6$ & $0.5 \pm 0.54$ \\
\hline Stiffness & $6.66 \pm 0.8$ & $0.66 \pm 0.51$ \\
\hline $\begin{array}{c}\text { Physical } \\
\text { Functioning }\end{array}$ & $60.5 \pm 1.8$ & $6.33 \pm 2.16$ \\
\hline $\begin{array}{c}\text { Total WOMAC } \\
\text { Score }\end{array}$ & $85.32 \pm 4.2$ & $7.49 \pm 3.21$ \\
\hline
\end{tabular}

San Diego, CA). The graphs 1-4 shows the value of pre and post induction and represents studies to be highly significance. $\mathrm{P}<0.0001$ (pre-therapy compared with post-therapy) was considered significant, Students ' $t$ ' test with Welch correction was performed to calculate $\mathrm{P}$ values. 
${ }^{* * *}$ Determined by Non Parametric T - Test

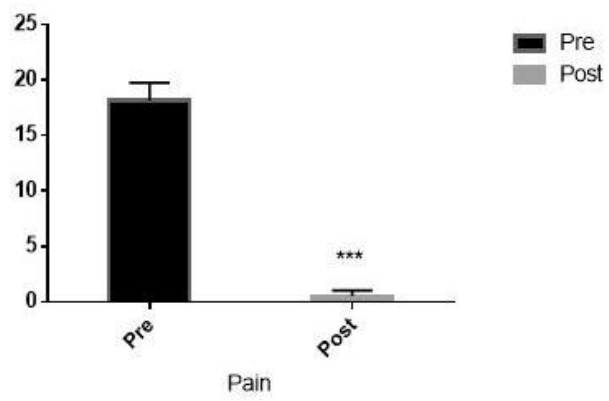

Graph 1: Statistical analysis of pain parameter of WOMAC index.

${ }^{s * 8}$ Determined by Non Parametric T - Test

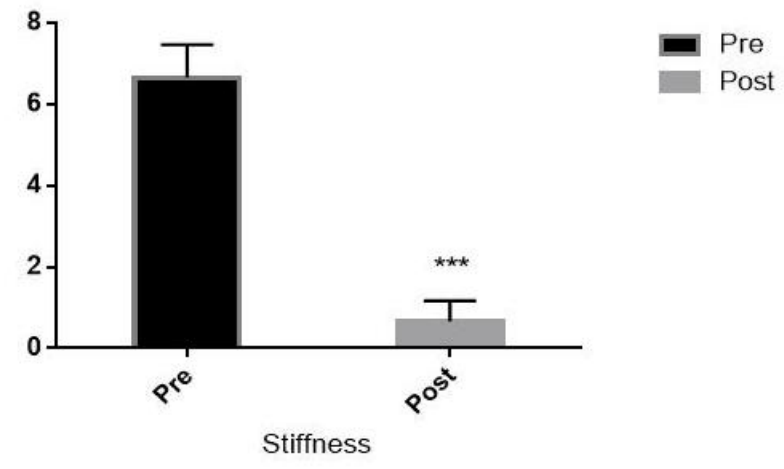

Graph 2: Statistical analysis of stiffness parameter of WOMAC index.

\section{Discussion}

In present study the mechanism of action for ADMSCs and PRP can be hypothesized. The PRP will reduce inflammation by secreting $\sim 150$ cytokines. The will provide an adherence site for MSCs. The MSCs will adhere to the lesion site depending upon the prevailing micro-environment in the knee joint. They will secrete cytokines, multiply, replicate and will form hyaline made embryonic like cartilage. Because of this very thin watery layer between tibia and femur the friction is reduced. This will help in pain relief, fine movements of knee joints.
${ }^{8 * 8}$ Determined by Non Parametric T - Test

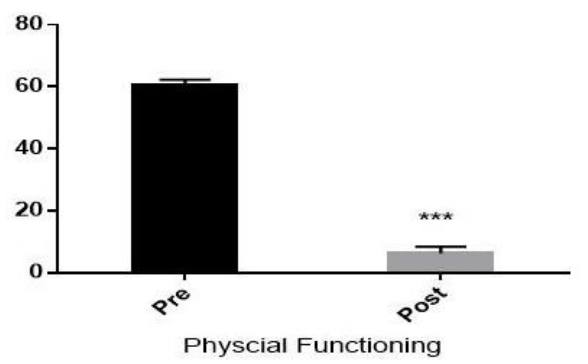

Pre Post

Graph 3: Statistical analysis of difficulty in physical functioning.

${ }^{* * *}$ Determined by Non Parametric T - Test

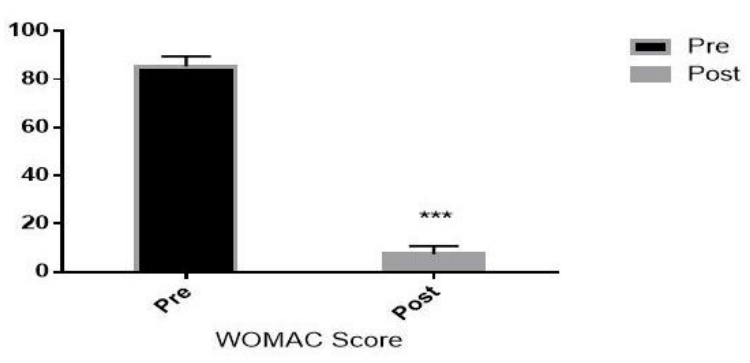

Graph 4: Statistical analysis of WOMAC score.

The subjective scoring with WOMAC score done after 12 months showed gradual decrease in WOMAC score which suggests that the patients have observed relief in pain, stiffness and physical limitations has improved. The total scoring of WOMAC is 96. All the patients before therapy were having minimum score of $85.32 \pm 4.2$. After the therapy there score reduced to $7.49 \pm 3.21$.

Fereydoun Davatchi et al. 2011 [12], reports 4 patients were treated with $30 \mathrm{ml}$ bone-marrow derived mesenchymal stem cells (BMMSCs) with dosage of 8-9 million cells after 4-5 weeks. The patients were assessed with VAS and X-ray after 0 , 6 and 12 months. The present pilot study includes 6 patients treated with adipose derived mesenchymal stem cells (ADMSCs). The percentage yield of MSCs is more in adipose tissue as compared to bone-marrow. The ADMSCs were harvested within 
2 weeks to attain the 2 million/ $\mathrm{kg}$ body weight dose. Keng Lin Wong et al. [13] reports that 28 patients were injected with BMMSCs with micro-fracture and high tibial osteotomy. The subjective scoring was done using International Knee Documentation Committee for 6 months, 1 year and 2 years. MRI scans performed 1 year after surgical intervention showed significantly better MOCART scores for the cell-recipient group. On contrary in the present study six patients were enrolled without any prior surgical interventions and controlled group.

The present study has limitations of no control group, effectiveness of different doses, optimum cell dose, small sample size to withdraw conclusion. The clinical assessment with MRI did not show significant change. The study needs to be monitored for more months. The inclusion parameters need to be changed with knee physiology in terms of alignment.

\section{Conclusion}

In nutshell, the results prove the therapeutic potential of mesenchymal stem cells as the pain is relieved, stiffness and physical functioning is improved after 12 months of the treatment. ADMSCs are safe as they did not have any immediate and systemic adverse events. Further study can be done with large sample size.

Acknowledgment: We acknowledge (late) Prof. Bharat Chattoo, M.S. University Baroda for rendering the use of laboratory. We are grateful to Dr. Udyayan Kachchi for negative marker studies.

Contributors: AM: Designing and monitoring study, manuscript editing; JT, PK: stem cell preparation, manuscript editing; SV: stem cell preparation, manuscript writing; $\mathrm{RV}$ : concept of the work, analysis of statistical data and its interpretation. AM will act as guarantor. All authors approved the final version of this manuscript.

Funding: None; Competing interests: None stated.

\section{References}

1. Arden N, Nevitt MC. Osteoarthritis: epidemiology. Best Pract Res Clin Rheumatol. 2006;20:3-25.

2. McAlindon TE, Bannuru RR, Sullivan MC, Arden NK, Berenbaum F, Bierma-Zeinstra SM, et al. OARSI guidelines for the non-surgical management of knee osteoarthritis. Osteoarthritis Cartilage. 2014;22:363.

3. Hattori H, Masuoka K, Sato M, Ishihara M, Asazuma T, Takase B, et al. Bone formation using human adipose tissue-derived stromal cells and a biodegradable scaffold. J Biomed Mater Res. 2006;1:230-239.

4. Pittenger MF, Mackay AM, Beck SC, Jaiswal RK, Douglas R, Mosca JD, et al. Multilineage potential of adult human mesenchymal stem cells. Science. 1999;2:143-147.

5. Patel DM, Shah J, Srivastava AS. Therapeutic potential of mesenchymal stem cells in regenerative medicine, Stem Cells International. 2013;2013:496218.

6. Zuk PA, Zhu M, Mizuno H, Huang J, Futrell W, Katz AJ, et al. Multilineage cells from human adipose tissue: Implications for cell-based therapies. Tissue Engineering. 2001;7:211-228.

7. Centeno CJ, Schultz JR, Cheever M, Robinson B, Freeman M, Marasco W. Safety and complications reporting on the re-implantation of culture-expanded mesenchymal stem cells using autologous platelet lysate technique, Curr Stem Cell Res Ther. 2010;5:81-93.

8. Carrillo-Mora P, González-Villalva A, MacíasHernández SI, Pineda-Villaseñor C. Platelet-rich plasma: a versatile tool for regenerative medicine? Cir Cir. 2013;81:70-77.

9. Chen HT, Lee MJ, Chen CH, Chuang SC, Chang LF, Ho ML, et al. Proliferation and differentiation potential of human adipose-derived mesenchymal stem cells isolated from elderly patients with osteoporotic fractures. J Cell Mol Med. 2012;16:582-593.

10. Pham PV, Hong-Thien Bui K, Ngo DQ, Vu NB, Truong NH, Lu-Chinh Phan N, et al. Activated plateletrich plasma improves adipose-derived stem cell transplantation efficiency in injured articular cartilage. Stem Cell Research \& Therapy. 2013;4:91-96.

11. Chen Z, Bai H, Pan YZ, Wang CB, Zhao Q, Hu XY, et al. X-ray induces autophagy in human mesenchymal stem cells. Zhonghua Xue Ye Xue Za Zhi. 2011;9:602605

12. Saatchi F, Abdollahi BS, Mohyeddin M, Shahram F, Nikbin B. Mesenchymal stem cell therapy for knee osteoarthritis, preliminary report of four patients. International Journal of Rheumatic Diseases. 2011;14:211-215.

13. Wong KL, Leng Lee KB, Tai BC, Law P, Lee EH, Hui JHP. Injectable cultured bone-marrow derived mesenchymal stem cells in varus knees with cartilage defects undergoing high tibial osteotomy: A prospective randomized controlled clinical trial with 2 years; follow up. Journal of Arthroscopy and Related Surgery 2013;29:2020-2028. 